\title{
Searching for hydrogen in Type Ib supernovae, and for helium and hydrogen in Type Ic supernovae
}

\author{
David Branch \\ Department of Physics and Astronomy, University of Oklahoma, \\ Nielsen Hall, Norman, OK 73019, USA
}

\begin{abstract}
Identifying the progenitors of Type Ib and TypeIc supernovae requires knowing, among other things, whether SNe Ib eject hydrogen, and whether SNe Ic eject helium, and perhaps even hydrogen. Recently it has become clear that some SNe Ib do eject hydrogen, and it may be that all SNe Ib do. Two arguments that have been made in the past that SNe Ic eject helium are difficult to confirm, but I discuss other possible evidence that SNe Ic eject helium, as well as hydrogen. If so, these elements extend to lower ejection velocities than in $\mathrm{SNe} \mathrm{Ib}$. The spectroscopic differences between $\mathrm{SNe} \mathrm{Ib}$ and SNe Ic may depend on the radial distributions of the helium and hydrogen as well as on the ejected masses of helium and hydrogen. We should consider the possibility that SNe Ic are more mixed up.
\end{abstract}

\section{Introduction}

Type Ia supernovae are thought to be nuclear-powered disruptions of accreting or merging white dwarfs. Most if not all other supernovae (SNe) are thought to involve expulsion of the envelopes of massive stars following the gravitational collapse of their highly evolved cores. Of these, TypeII SNe have conspicuous features due to hydrogen in their optical spectra. Type Ib SNe lack conspicuous hydrogen features but have conspicuous He I features during their photospheric phase. In the spectra of TypeIc SNe, neither hydrogen nor He I features are conspicuous. For reviews of SN classification see Filippenko (1997) and Turatto (2002).

The optical spectrum of a typical SN II evolves from a nearly featureless continuum when the temperature is high to one that contains mainly hydrogen features and then gradually develops features of lower excitation due to CaII, Fe II, O I, NaI, and TiII, plus BaII and Sr II if the temperature gets sufficiently low. Apart from the defining differences involving the hydrogen and $\mathrm{He}$ I features, the spectra of $\mathrm{SNe} \mathrm{Ib}$ and $\mathrm{SNe}$ Ic evolve in much the same way.

Among the questions that those who make explosion models of $\mathrm{SNe} \mathrm{Ib}$ and SNe Ic would like spectroscopists to answer include two very basic ones. Do SNe Ib eject any hydrogen? And do SNe Ic eject any helium - and perhaps even some hydrogen? I will be able to provide only partial and tentative answers to these questions - but I should at least be able to explain why they are so hard to answer. 


\section{Direct analysis of supernova spectra}

First we must say something about the analysis of SN spectra. One nice thing is that the simple explosion velocity law - velocity proportional to radius - ordinarily is an excellent approximation. One big problem is that ejection velocities usually are high, so spectral features are blended and the amount of information that can be obtained by analyzing the spectra with a ruler is limited. Consequently, much use is made of synthetic spectra. The use of synthetic spectra for SNe has been reviewed by Branch, Baron \& Jeffery (2002). Detailed and physically self-consistent non-LTE synthetic spectra can and have been carried out with very complex codes, notably the PHOENIX code, but so far nearly all such work has been for SNeIa and SNeII. At the other extreme, of simplicity, the fast parameterized SYNOW code is useful for establishing line identifications and placing constraints on the velocity intervals in which the line-contributing ions are detected, We refer to this process as 'direct analysis' to distinguish it from detailed spectrum synthesis with more complicated codes. The synthetic spectra shown in this article have been generated with sYNOw.

SYNOW assumes spherical symmetry, velocity proportional to radius, and a sharp photosphere that emits a blackbody continuum. Line formation, treated in the Sobolev approximation, occurs only by resonance scattering of photons from the photosphere. The effects of multiple scattering (line blending) on the line source functions and the emergent spectrum are treated exactly, within the context of the model and the Sobolev approximation. In the synthetic spectra shown in this article, line optical depths are taken to decrease as $v^{-8}$. For each ion whose lines are introduced, the optical depth of a 'reference line' at the photosphere is a fitting parameter, and the optical depths of the other lines of the ion are determined by assuming LTE level populations at some excitation temperature $T_{\text {exc }}$, which for the synthetic spectra of this article is $7000 \mathrm{~K}$. Oscillator strengths are from Kurucz (1993). Line formation ordinarily takes place immediately above the photosphere, i.e., the lower boundary of the line-forming layer has the velocity at the photosphere, $v_{\text {phot }}$. But sometimes it is appropriate to 'detach' the lines of an ion by allowing line formation only above some detachment velocity that exceeds $v_{\text {phot }}$.

An undetached resonance-scattering line has a P-Cygni profile consisting of a rounded emission component that peaks at the rest wavelength, and an absorption component whose minimum is blue-shifted by the amount that corresponds to $v_{\text {phot }}$ (or more if the line is strong). A detached resonance-scattering line also has a P-Cygni profile but the emission component has a flat top, and the absorption minimum is blue-shifted by the amount that corresponds to the detachment velocity. The absorption component of a detached line is deeper than that of an undetached line that has the same maximum Sobolev optical depth (Jeffery \& Branch 1990; Branch et al. 2002).

\section{SNe IIb}

Before discussing SNeIb we must mention SNeIIb - events in which hydrogen features are conspicuous at early times but later become weak or undetectable. The first well observed example was SN 1993J in M 81 (Matheson et al. 2000 and numerous references therein). Conspicuous hydrogen features were present at early times, but conspicuous He I features began to appear around 25 days after 
explosion; later the hydrogen features became weak. The minimum velocity of the hydrogen-containing layer was about $9000 \mathrm{~km} \mathrm{~s}^{-1}$. Most estimates of the mass of this layer have been in the range 0.1 to $0.6 \mathrm{M}_{\odot}$; the hydrogen mass was lower because much of the mass of this layer probably was in the form of helium. In SN 1996cb, the second well observed SN IIb (Qiu et al. 1999), He I features appeared earlier, before maximum light, but a deep $\mathrm{H} \alpha$ absorption persisted throughout the photospheric phase. The minimum velocity and mass of the hydrogen-containing layer were about $10000 \mathrm{~km} \mathrm{~s}^{-1}$ and perhaps $\sim 0.1 \mathrm{M}_{\odot}$ (Deng, Qiu \& Hu 2002).

\section{Hydrogen in SNe Ib?}

Until recently not much work had been done on searching for hydrogen in SNe Ib. Branch (1972) considered $\mathrm{H} \alpha$, as well as the strongest optical tine of $\mathrm{Ne}$ I $\lambda 6402$, as possible identifications of a deep absorption in SN 1954A. Wheeler et al. (1994) tentatively attributed a weak absorption in SN $1983 \mathrm{~N}$ and SN 1984L to H $\alpha$, as did Deng et al. (2000) in SN 1999dn. An opportunity to study this issue more systematically was presented by Matheson et al. (2001, hereafter M01) who published spectra of a number of SNe Ib (and SNe Ic) that were observed at the Lick Observatory during the 1990s.

Recently the SYNOW code has been used to study the spectra of $11 \mathrm{SNe} \mathrm{Ib}$ selected on the basis of having deep optical He I absorptions (Branch et al. 2002, hereafter B02). As had been emphasized by M01, from whom much of the data of the B02 sample were taken, in most respects the spectra of these events were quite homogeneous. The velocity at the photosphere, as inferred from Fe II features near $5000 \AA$, decreased smoothly from about $11000 \mathrm{~km} \mathrm{~s}^{-1}$ at the time of maximum light to about $5000 \mathrm{~km} \mathrm{~s}^{-1} 50$ days later, with little scatter. In post-maximum spectra the HeI absorptions generally were detached, with the detachment velocity ending up at about $7000 \mathrm{~km} \mathrm{~s}^{-1}$.

Two events in the B02 sample, SN 1999di and SN 2000H, differed from the others by containing deep, persistent absorptions near $6300 \AA$ that resembled the deep $\mathrm{H} \alpha$ absorption in the Type IIb SN 1996cb. It is safe to identify the $6300 \AA$ absorptions in these two SNeIb as $\mathrm{H} \alpha$, detached at 12000 or $13000 \mathrm{~km} \mathrm{~s}^{-1}$, because there is no plausible alternative identification that would not entail unwanted features in the spectrum, and because weak absorptions consistent with $\mathrm{H} \beta$ could be seen. SN 1954A, for which of course only photographic spectra are available, appears to have been similar. I will refer to these events as 'deep-H $\alpha$ ' $\mathrm{SNe}$ Ib. They are classified as Type Ib because when they were first observed the presence of hydrogen features was not obvious, even though their $\mathrm{H} \alpha$ absorptions were deep. The explanation, illustrated in figure 24 of B02, is that because the hydrogen lines were detached, the $\mathrm{H} \alpha$ emission components that we are accustomed to seeing in SNe II were flat and therefore inconspicuous, and the deep $\mathrm{H} \alpha$ absorptions were produced with sufficiently small $\mathrm{H} \alpha$ optical depths that the other Balmer lines were too weak to be conspicuous. (The Sobolev optical depth of $\mathrm{H} \beta$ is lower than that of $\mathrm{H} \alpha$ by a factor of 7.) If these events had been observed earlier their hydrogen features might have been stronger, undetached, and conspicuous, as in the earliest spectra of SN $1996 \mathrm{cb}$, in which case these events would have been classified as SNeIIb. 


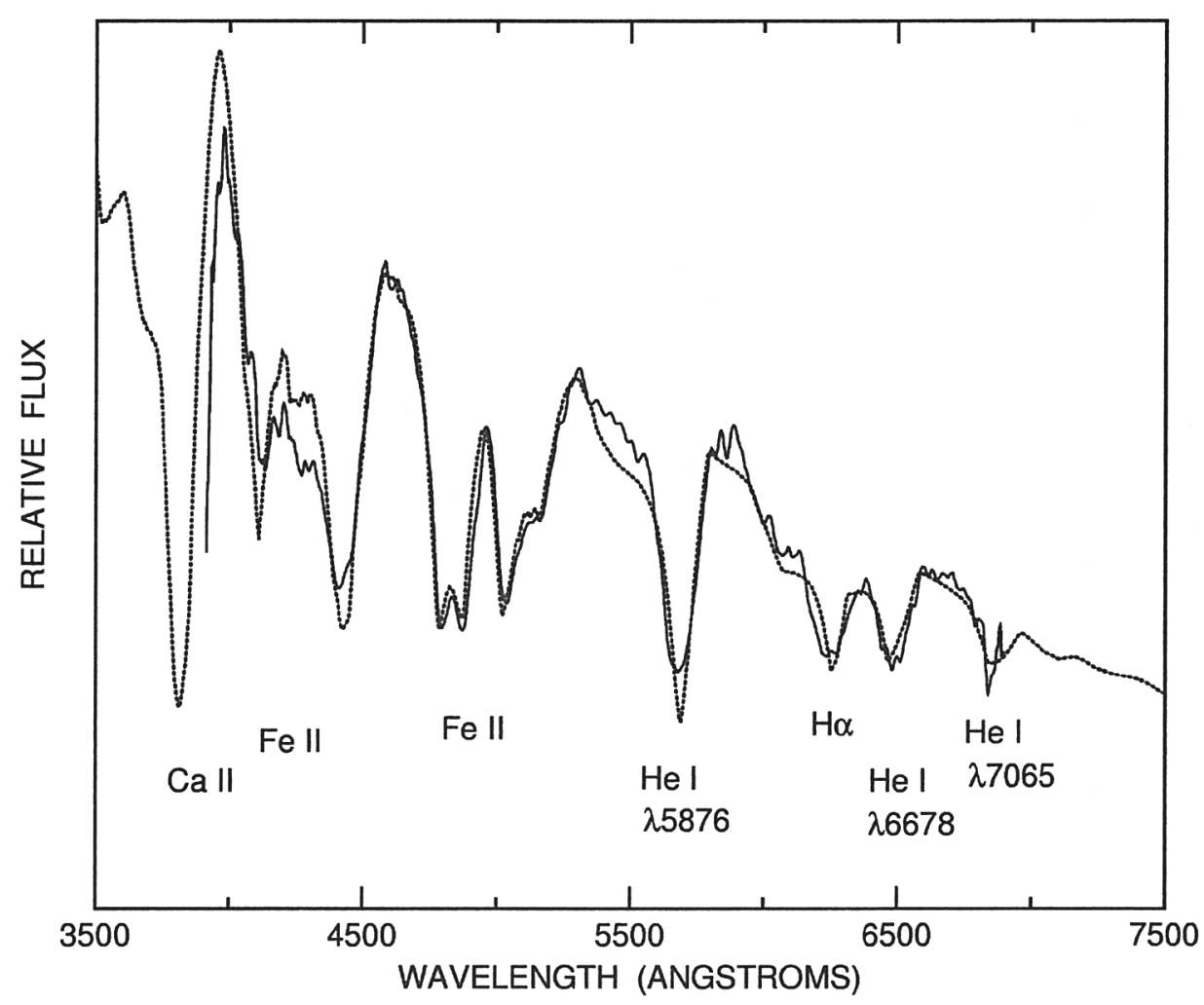

Figure 1. A spectrum of the Type Ib SN 1984L obtained by Harkness et al. (1987) 9 days after maximum brightness (solid line) is compared with a synthetic spectrum (dotted line) that has $v_{\text {phot }}=9000 \mathrm{~km} \mathrm{~s}^{-1}$ and contains lines of H, HeI, CaII, Sc II, and Fe II. The hydrogen lines are detached at $15000 \mathrm{~km} \mathrm{~s}^{-1}$. In this and subsequent figures the flux is per unit wavelength interval and its scale is arbitrary. Figure adapted from Branch et al. (2002).

Among the eight other events of the B02 sample, four (SNe 1983N, 1984L, $1996 \mathrm{~N}$, and $1999 \mathrm{dn}$ ) were first observed earlier than 10 days after maximum light and they also had absorptions near $6300 \AA$, but less deep than in the deep- $\mathrm{H} \alpha$ events. I will refer to these as typical SNe Ib. In Figure 1 a spectrum of SN 1984L is compared with a synthetic spectrum. B02 attributed the $6300 \AA$ absorption in typical SNe Ib to $\mathrm{H} \alpha$, detached at $18000 \mathrm{~km} \mathrm{~s}^{-1}$ in the earliest available spectrum and at 12000 or $13000 \mathrm{~km} \mathrm{~s}^{-1}$ when it was last observed. The identification was tentative, however, because $\mathrm{H} \beta$ could not be seen, and there are other possible line identifications for the $6300 \AA$ absorption. The strongest optical line of $C_{\text {II }}$ $\lambda 6580$, would need to be detached only $800 \mathrm{~km} \mathrm{~s}^{-1}$ more than $\mathrm{H} \alpha$, but highly detached $\mathrm{C}$ II lines would be surprising in $\mathrm{SNeIb}$. The strongest optical line of Si II $\lambda 6355$, would need to be blue-shifted $9500 \mathrm{~km} \mathrm{~s}^{-1}$ less than $\mathrm{H} \alpha$, which is a problem because it would require the Si II feature to form in deeper layers than the $\mathrm{Fe}$ II features. A more plausible alternative to $\mathrm{H} \alpha$ is $\mathrm{Ne}$ I $\lambda 6402$, which would need to be blue-shifted $7400 \mathrm{~km} \mathrm{~s}^{-1}$ less than $\mathrm{H} \alpha$; this would put it right at the 


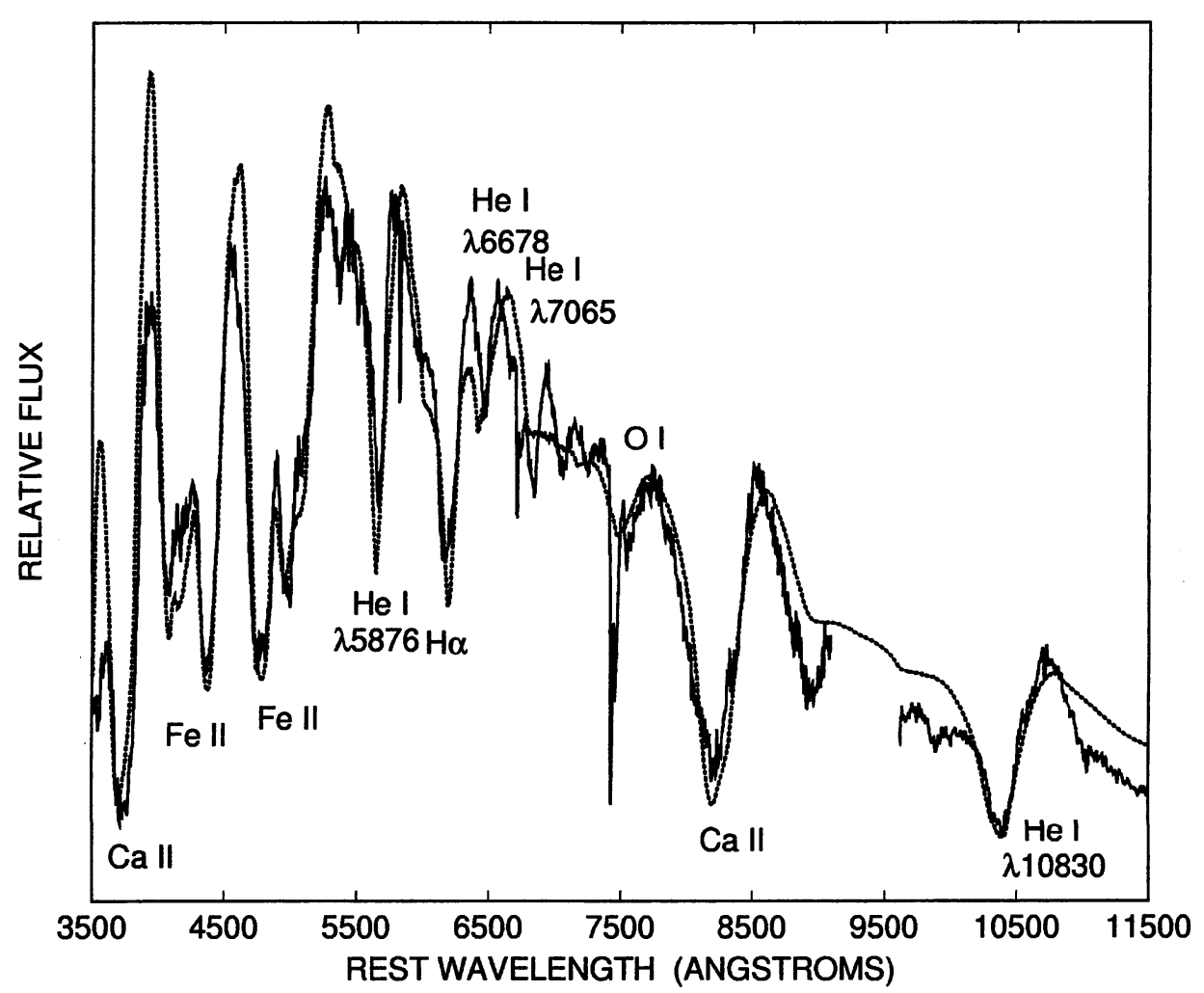

Figure 2. A spectrum of the Type Ib SN 1999ex obtained by Hamuy et al. (2002) 4 days after maximum brightness (solid line) is compared with a synthetic spectrum (dotted line) that has $v_{\text {phot }}=9000 \mathrm{~km} \mathrm{~s}^{-1}$ and contains lines of H, HeI, O I, CaII, and Fe II. The hydrogen lines are detached at $15000 \mathrm{~km} \mathrm{~s}^{-1}$.

same velocity as the Fe II lines. The Ne I lines would need to be nonthermally excited, as are the HeI lines of SNeIb. When Fe II features become strong they also can produce a feature that could be mistaken for highly blue-shifted $\mathrm{H} \alpha$ (see below), but in these four $\mathrm{SNe}$ Ib the Fe II features were not strong enough around the time of maximum. The best choices for the $6300 \AA$ absorption in typical SNe Ib seem to be detached $\mathrm{H} \alpha$ or undetached $\mathrm{Ne}$ I $\lambda 6402$.

Only very recently has it become clear that some events have optical He I absorptions that are definite but not as deep as in typical SNe Ib. I will refer to these as 'shallow-helium' SNe Ib. One of these, SN 1991D (Benetti et al. 2002), deviates from typical SNe Ib in other respects too. It was exceptionally luminous for a SN Ib; 10 days after maximum light the velocity at the photosphere inferred from the FeII features was only $5000 \mathrm{~km} \mathrm{~s}^{-1}$ as opposed to $8000 \mathrm{~km} \mathrm{~s}^{-1}$ for typical $\mathrm{SNeIb}$; and at the same epoch the He I lines were not detached from the $5000 \mathrm{~km} \mathrm{~s}^{-1}$ photosphere whereas in typical SNeIb they are detached at about $10000 \mathrm{~km} \mathrm{~s}^{-1}$. SN 1991D contained a weak $6300 \AA$ absorption that can be accounted for by $\mathrm{H} \alpha$ detached at $12000 \mathrm{~km} \mathrm{~s}^{-1}$, but in SYNOW synthetic spectra 
not only did $\mathrm{Ne}$ I $\lambda 6402$ provide a nice fit to this feature, other $\mathrm{Ne}$ I lines did more good than harm (figure 3 of Benetti et al.). Ne I $\lambda 6402$ appears to be a serious alternative to $\mathrm{H} \alpha$ in SN 1991D.

Hamuy et al. (2002) have presented spectra of another shallow-helium SN Ib, SN 1999 ex. In this case the Fe II velocity evolution does conform to that of typical SNe Ib. The depth of the $6300 \AA$ absorption of SN 1999ex was in between those of typical and deep-H $\alpha$ SNeIb. Hamuy et al. labelled the $6300 \AA$ absorption as Si II but this identification encounters the problem mentioned above. In Figure 2 a spectrum of SN 1999ex is compared with a synthetic spectrum in which the $6300 \AA$ absorption is produced by $\mathrm{H} \alpha$, detached at $15000 \mathrm{~km} \mathrm{~s}^{-1}$. Undetached Ne I $\lambda 6402$ also can fit the $6300 \AA$ absorption fairly well but because it needs to be rather strong the other $\mathrm{Ne}$ I lines cause problems. $\mathrm{H} \alpha$ appears to be the most likely identification in SN 1999ex.

\section{Helium in $\mathrm{SNeIc}$ ?}

In the past, two lines of evidence have been offered for the presence of He I features in SNe Ic, but both are difficult to confirm. First, in SN 1994I, the best observed SN Ic (other than hypernovae), Filippenko et al. (1995) observed a strong absorption near $1 \mu \mathrm{m}$ that was attributed to He I $\lambda 10830$. However, when we (Millard et al. 1999) carried out a SYNOw analysis of SN 1994I, we were unable to account for the bulk of the $1 \mu \mathrm{m}$ absorption with $\lambda 10830$ without also producing optical He I features that were too strong. We concluded that the $1 \mu \mathrm{m}$ absorption may have been produced at least in part by $\mathrm{C}_{\mathrm{I}} \lambda 10695$ and/or blends of Si I lines. Baron et al. (1999) carried out detailed spectrum calculations with the PHOENIX code and also had problems accounting for the $1 \mu \mathrm{m}$ feature in terms of $\lambda 10830$ alone.

The observations of the Type Ib SN 1999ex by Hamuy et al. (2002) clarify this issue. Hamuy et al. obtained good infrared spectra of SN 1999ex, and as expected from the presence of definite optical He I features, the infrared spectra contained P-Cygni profiles due to He I $\lambda 10830$ and He I $\lambda 20581$. (In LTE the Sobolev optical depth of He I $\lambda 10830$ exceeds that of He I $\lambda 5876$ by a factor of 7 at $10000 \mathrm{~K}$ and a factor of 4.5 at $15000 \mathrm{~K}$.) However, even though SN $1999 \mathrm{ex}$ had definite optical He I lines, its $\lambda 10830$ feature was weaker than the $1 \mu \mathrm{m}$ feature in SN 1994I, which had less distinct, if any, optical He I features. It is interesting that in SN 1999ex, unlike in SN 1994I, a SYNOW synthetic spectrum that fits the $\lambda 10830$ feature also fits the optical He I features reasonably well. This supports the suspicion that the $1 \mu \mathrm{m}$ absorption in SN 1994I was not mainly due to $\mathrm{He}$ I $\lambda 10830$ - and if it wasn't mainly due to He I then it doesn't provide convincing evidence for the presence of helium at all.

The second line of evidence for He I features in SNeIc has been the identification of weak absorptions in optical spectra with high-velocity He I lines, detached at about $17000 \mathrm{~km} \mathrm{~s}^{-1}$ (Clocchiatti et al. 1996). However, M01 reexamined the evidence and were unable to confirm these identifications. One problem was that the absorption attributed to detached He I $\lambda 6678$ usually is substantially more blue-shifted than those attributed to $\lambda 5876$ and $\lambda 7065$. M01 also mentioned that the typical Type Ib SN 1999dn, whose deep He I absorptions were at low velocity, contained a weak absorption like the one that Clocchiatti et al. attributed to high-velocity $\lambda 5876$ in SNeIc. In fact, that feature often 


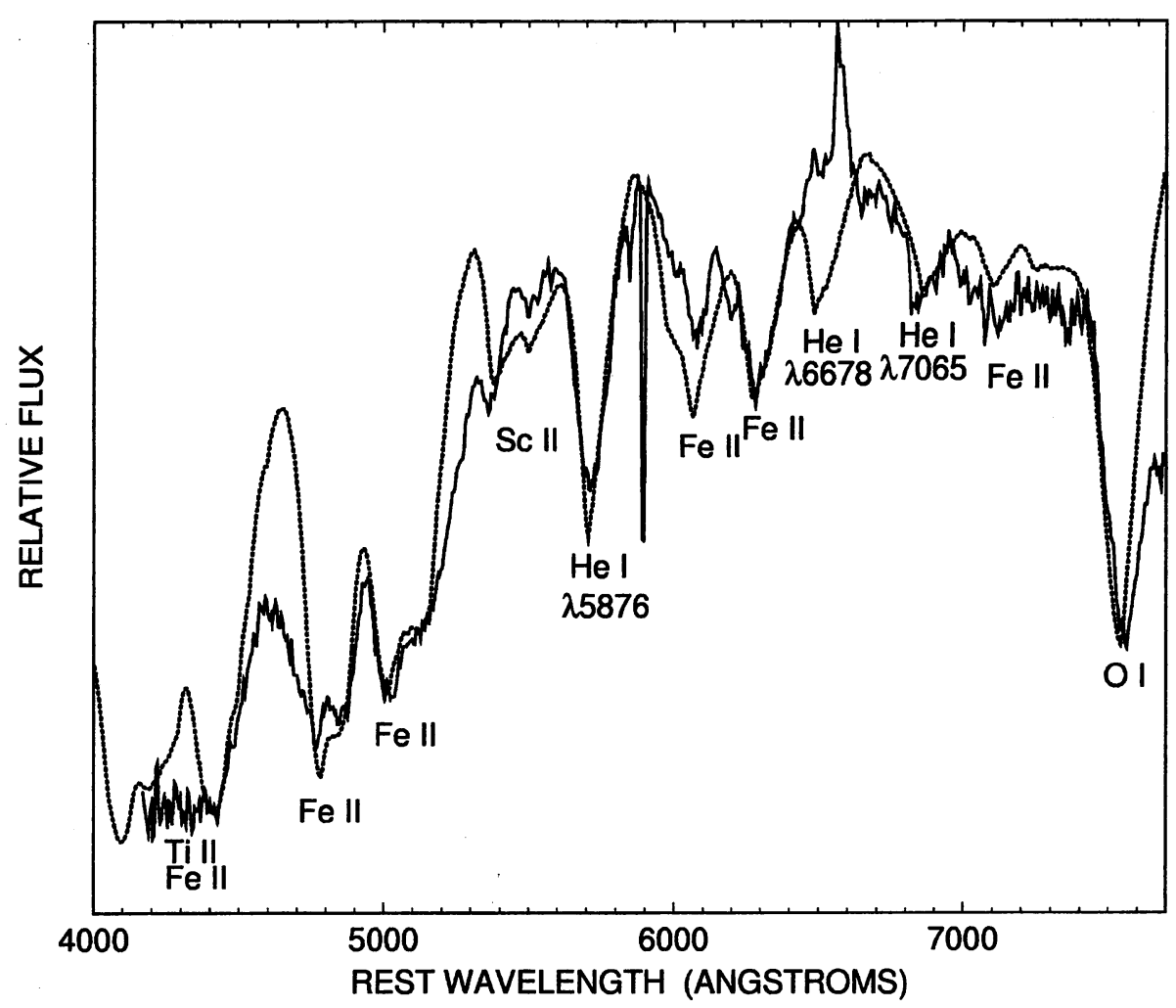

Figure 3. A spectrum of the Type Ic SN 1990B obtained by Matheson et al. (2001) 5 days after maximum brightness (solid line) is compared with a synthetic spectrum (dotted line) that has $v_{\text {phot }}=9000 \mathrm{~km} \mathrm{~s}^{-1}$ and contains lines of He I, O I, Ca II, Sc II, Ti II, and Fe II. The narrow peak at $6563 \AA$ is $\mathrm{H} \alpha$ emission from an H II region and the narrow absorption at $5892 \AA$ is due to interstellar $\mathrm{NaI}$.

appears in spectra of core-collapse SNe, including the Type II SN 1987A (Jeffery $\&$ Branch 1990), when the temperature is low enough for lines of singly ionized iron-peak elements to be strong. It is produced by a group of Sc II lines from $\lambda 5641$ to $\lambda 5684$.

Being unpersuaded by the first two lines of evidence for He I in SNeIc, we consider a third. After failing to confirm high-velocity He I absorptions in SNe Ic, M01 mentioned that in the Type Ic SN 1990B, if He I absorptions were present at all, they were at low velocity. Clocchiatti et al. (2001) also considered the possibility of low-velocity He I in SN 1990B. In Figure 3 a spectrum of SN 1990B is compared with a synthetic spectrum that contains undetached He I lines. Note that because Fe II features are quite strong, they account nicely for the $6300 \AA$ absorption. He I $\lambda 5876$ accounts for an observed absorption, but of course that observed feature could be NaI instead. More important is that the observed absorption near $6800 \AA$ is accounted for by undetached He I $\lambda 7065$, and I'm not aware of any good alternative for undetached $\lambda 7065$; neither a line of similar 
wavelength nor a line of longer wavelength that could be detached and mistaken for undetached $\lambda 7065$. An obvious problem is that there is no counterpart in the observed spectrum for the synthetic absorption produced by $\lambda 6678$, but this is not necessarily fatal for He I. First, M01 showed that in SNeIb $\lambda 6678$ gradually weakens relative to $\lambda 5876$ and $\lambda 7065$; this is to be expected on the grounds of non-LTE calculations (Lucy 1991; Swartz et al. 1993) because $\lambda 6678$ is a singlet transition while $\lambda 5876$ and $\lambda 7065$ are triplets, and the singlet resonance transition to the ground state becomes less opaque as the ejecta density decreases through expansion. It follows that the use of LTE excitation in SYNOw can overestimate the strength of $\lambda 6678$ relative to $\lambda 5876$ and $\lambda 7065$. Second, in SN 1990B the $\lambda 6678$ absorption would be partially filled in by the $\mathrm{H}$ II-region $\mathrm{H} \alpha$ emission seen in the spectrum, and possibly also by $\mathrm{H} \alpha$ emission from SN 1990B itself (see Section 6).

Another reason to take low-velocity $\mathrm{He} \mathrm{I}$ in $\mathrm{SNe}$ Ic seriously is that the early spectra of SN 1994I contained an absorption feature that we (Millard et al. 1999) attributed to $\mathrm{NaI} \lambda 5892$. But at these early times the color temperature was high - $\sim 17000 \mathrm{~K}$ in the spectrum obtained four days before maximum brightness. The presence of $\mathrm{NaI}$ absorption at such a high temperature is doubtful. In SN 1987A, for example, Na I began to appear only when the color temperature fell to about $6000 \mathrm{~K}$ (Jeffery \& Branch 1990). In the early spectra of SN 1994I even the CaII absorptions were not yet very strong. The observed feature may have been produced by undetached He I $\lambda 5876$ rather than NaI.

During the course of an ongoing comparative study of spectra of SNeIc, similar to that of B02 for SNeIb, it has become clear that absorptions that can be attributed to undetached HeI $\lambda 5876$ and $\lambda 7065$ are common in the early spectra of SNeIc, and sometimes a weak absorption consistent with $\lambda 6678$ also is present.

\section{Hydrogen in SNe Ic?}

In the past, two lines of evidence for the presence of $\mathrm{H} \alpha$ in SNe Ic also have been presented, but again confirmation is difficult. Filippenko, Porter, \& Sargent (1990) and Jeffery et al. (1991) tentatively attributed a weak absorption near $6360 \AA$ in SN $1987 \mathrm{M}$ to $\mathrm{H} \alpha$, but as emphasized by Wheeler et al. (1994) the required blue-shift of $\mathrm{H} \alpha$ was suspiciously low compared to those of other lines in the same spectrum. In any case, the absorption in question is unusual, perhaps even unique so far; it is not the same as the broader feature that is commonly referred to as the $6300 \AA$ absorption, which ordinarily appears at a wavelength roughly consistent with undetached $\mathrm{Ne}$ I $\lambda 6402$ (whether $\mathrm{Ne} I$ is the right identification or not). The $6360 \AA$ absorption in SN $1987 \mathrm{M}$ is farther to the red.

More intriguing is that Filippenko $(1988,1992)$ pointed out that SNe Ic have a broad emission peak near the wavelength of $\mathrm{H} \alpha$. We must be careful about attributing this feature to $\mathrm{H} \alpha$ emission when the Fe II features are strong - but it appeared even in the pre-maximum spectra of SN 1994I, when the Fe II features were not yet strong. It is indeed tempting to attribute this feature to $\mathrm{H} \alpha$. The $\mathrm{H} \alpha$ would need to be in net emission (as it often is in SNeII), and to produce the rounded emission peak the $\mathrm{H} \alpha$ would need to be undetached. 


\section{Discussion}

So - do SNe Ib eject hydrogen? Yes, the deep-H $\alpha$ events certainly do; they appear to be closely related to SNe IIb. In fact, the differences between SNeIIb and the deep-H $\alpha$ SNe Ib can be so small that the classification of some events probably depends on how early the first spectrum is obtained ${ }^{1}$.

Whether typical SNeIb eject hydrogen is much more difficult to decide. Given the strong similarities between the deep- $\mathrm{H} \alpha$ and typical SNe Ib (B02), the most natural assumption would seem to be that the $6300 \AA$ absorption in typical $\mathrm{SNe} \mathrm{Ib}$ also is produced by $\mathrm{H} \alpha$. This is supported by the probable presence of intermediate-strength $6300 \AA$ absorption in the shallow-helium SN 1999ex. On the other hand, nonthermally excited NeI lines are not implausible, and because $\mathrm{NeI} \lambda 6402$ would not need to be detached it would require one fewer free parameter (the detachment velocity of $\mathrm{H} \alpha$ ). The $6300 \AA$ absorption tends to drift redward with time like the other features; this may seem to favor undetached NeI, but it is not decisive since the detached He I lines in SNe Ib also drift to the red. (B02 offer an explanation.) How are we to decide about the $\mathrm{H} \alpha$ identification? The most direct confirmation would be to detect Paschen-alpha ( $\mathrm{P} \alpha \lambda 18751)$, but in LTE its Sobolev optical depth is lower than that of $\mathrm{H} \alpha$ by a factor of 34 at $5000 \mathrm{~K}$ and 3.4 at $10000 \mathrm{~K}$, so this may not work. A larger sample of high quality optical spectra of typical $\mathrm{SNe} \mathrm{Ib}$ will allow the following test to be applied: if the $6300 \AA$ feature always is consistent with undetached NeI, then it probably is NeI, because it would be hard to believe that hydrogen always is detached by the same amount with respect to the velocity at the photosphere. The current data are inconclusive on this point.

Do SNe Ic eject helium? As we have seen, the $1 \mu \mathrm{m}$ feature in SN 1994I does not provide convincing evidence for He I $\lambda 10830$, and the presence of weak optical absorptions with highly detached He I lines is unlikely. However, it may be that the optical spectra contain undetached He I lines. How are we to decide? Since He I $\lambda 10830$ can be confused with lines of other ions, the most direct test might be to look for $\lambda 20581$, which in LTE has about the same Sobolev optical depth as $\lambda 10830$ above $10000 \mathrm{~K}$. (But be careful $-\lambda 20581$ is a singlet transition.) Infrared spectra also could be used to sort out the contributions of $\mathrm{CI}$ and $\mathrm{Si}$ I to the $1 \mu \mathrm{m}$ feature because both ions have other strong lines that should be detectable (figure 12 of Millard et al. 1999).

Do SNe Ic eject hydrogen? Identifying the weak $6360 \AA$ absorption with $\mathrm{H} \alpha$ in SN 1987M may be questionable, but the possibility of emission near the rest wavelength of $\mathrm{H} \alpha$ looks interesting. The hydrogen would need to be undetached. This, together with the possibility that SNeIc contain undetached He I lines, means that if SNe Ic do eject helium and hydrogen then these elements extend to lower ejection velocities than in SNeIb. The appearance of the hydrogen and $\mathrm{He}$ I features in SNeIb and SNe Ic may be affected by the radial distributions of the helium and hydrogen in addition to the total ejected masses of helium and hydrogen. We should consider the possibility that the composition structures of SNe Ic are more mixed up than those of SNeIb.

\footnotetext{
${ }^{1}$ In the nomenclature of Clocchiatti \& Wheeler (1997) the deep-H $\alpha$ events could be included among the 'transition supernovae', SNe IIt.
} 
This discussion of line identifications has been based on calculations carried out with the simple SYNOw code. Detailed non-LTE calculations for a grid of explosion models for SNe Ib and SNeIc are needed, especially to test the possibilities of nonthermally excited Ne I lines in SNeIb (and SNeIc), and of rounded $\mathrm{H} \alpha$ emission in SNeIc. It also is needed in order to estimate, or place upper limits on, the masses of helium and hydrogen in SNeIb and SNe Ic.

Clocchiatti et al. (1997) have emphasized that light-curve shapes imply significant differences in the ejected masses of supernovae of the same spectral type. This, together with our uncertainty about the composition structures of the ejected matter, and our still scanty knowledge of the relative occurrence frequencies of the various kinds of SNeIb and SNe Ic, means that there is much work to be done before we will be able to match the various kinds of $\mathrm{SNe} \mathrm{Ib}$ and $\mathrm{SNe}$ Ic with their stellar progenitors in a detailed way.

Acknowledgments. I am grateful to Tom Matheson and Mario Hamuy for providing spectra; to them, Eddie Baron, Jinsong Deng, Chris Gerardy, and David Jeffery for comments on the manuscript; and to members of the University of Oklahoma supernova group for many discussions. This work has been supported by NSF grant AST-9986965.

\section{References}

Baron, E., Branch, D., Hauschildt, P.H., Filippenko, A.V., Kirshner, R.P. 1999, ApJ 527, 739

Benetti, S., Branch, D., Turatto, M., et al. 2002, MNRAS 336, 91

Branch, D. 1972, A\&A 16, 247

Branch, D., Benetti, S., Kasen, D., et al. 2002, ApJ 566, 1005 (B02)

Branch, D., Baron, E., Jeffery, D.J. 2002, in: K.W. Weiler (ed.), Supernovae and Gamma-Ray Bursts (Berlin: Springer), in press (astro-ph/0111573)

Clocchiatti, A., Wheeler, J.C., Phillips, M.M., et al. 1997, ApJ 483, 675

Clocchiatti, A., Suntzeff, N.B., Phillips, M.M., et al. 2001, ApJ 553, 886

Clocchiatti, A., Wheeler, J.C. 1997, ApJ 491, 375

Clocchiatti, A., Wheeler, J.C., Brotherton, M.S., Cochran, A.L., Wills, D., Barker, E.S., Turatto, M. 1996, ApJ 462, 462

Deng, J., Qiu, Y.L., Hu, J. 2002, ApJ submitted (astro-ph/0106404)

Deng, J., Qiu, Y.L., Hu, J., Hatano, K., Branch, D. 2000, ApJ 540, 452

Filippenko, A.V. 1988, AJ 96, 1941

Filippenko, A.V. 1992, ApJ (Letters) 384, L37

Filippenko, A.V. 1997, Ann. Review Astron. Astrophys. 35309

Filippenko, A.V., Barth, A.A., Matheson, T., et al. 1995, ApJ (Letters) 450, L11

Filippenko, A.V., Porter, A., Sargent, W.L.W. 1990, AJ 100, 1575

Hamuy, M., Maza, J., Pinto, P.A., et al. 2002, AJ 124, 417

Harkness, R.P., Wheeler, J.C., Margon, B., et al. 1987, ApJ 317, 355

Jeffery, D.J., Branch, D. 1990, in: J.C. Wheeler, T. Piran \& S. Weinberg (eds.), Supernovae, Sixth Jerusalem Winter School for Theoretical Physics (Singapore: World Scientific), p. 149

Jeffery, D.J., Branch, D., Filippenko, A.V., Nomoto, K. 1991, ApJ 317, 717

Kurucz, R.L. 1993, CD-ROM 1, Atomic Data for Opacity Calculations (Cambridge: SAO) 
Lucy, L.B. 1991, ApJ 383, 308

Matheson, T., Filippenko, A.V., Bart, A.A., Ho, L.C., et al. 2000, AJ 120, 1487

Matheson, T., Filippenko, A.V., Li, W., Leonard, D.C., Shields, J.C. 2001, AJ 121, 1648 (M01)

Millard, J., Branch, D., Baron, E., et al. 1999, ApJ 527, 746

Qiu, Y., Li, W., Qiao, Q, Hu, J. 1999, AJ 117, 736

Swartz, D.A., Filippenko, A.V., Nomoto, K., Wheeler, J.C. 1993, ApJ 411, 313

Turatto, M. 2002, in: K.W. Weiler (ed.), Supernovae and Gamma-Ray Bursts (Berlin: Springer), in press

Wheeler, J.C., Harkness, R.P., Clocchiati, A., Benetti, S., Brotherton, M.S., DePoy, D.L., Elias, J. 1994, ApJ (Letters) 436, L135

\section{Discussion}

KUDTRITZKI: When you include the detached $\mathrm{H} \alpha$-fits, you certainly obtain a very good fit of the blue-shifted line absorption of the suspicious feature. However, the fit on the red-ward side becomes worse. What do you think about this?

BRANCH: The poorer fit on the red side is caused by the simple but unrealistic assumption that the line optical depth increases discontinuously from zero at the detachment velocity. A smooth increase would lead to a nicer fit.

MAEDER: Do you think that in future you may be able to identify the kind of progenitors which is to be assigned to SN Ib and SN Ic? Are they WN or WC subtypes? Could CNO abundances be helpful in this identification?

BRANCH: Yes, but the detailed matching of the various kinds of SNeIb and SNeIc will require detailed spectrum calculations for detailed hydrodynamical models. We can see oxygen and perhaps carbon lines in SNeIb and SNeIc, but nitrogen is hard to see.

WALBORN: (i) Can He I $\lambda 3889$ be used as a diagnostic? It is a very prominent feature in peculiar OB spectra, that behaves similarly to $\lambda 10830$. (ii) What progenitor stellar types do your results favor? I believe there is evidence that SN 1993J was a red supergiant?

BrANCH: In supernovae, He I $\lambda 3889$ is too blended with other lines, including strong Ca II $\lambda 3933$. Yes, the progenitor of SN 1993J appears to have been a K-type supergiant, but the ejected hydrogen mass was on the order of $0.1 \mathrm{M}_{\odot}$. In SNeIb the ejected hydrogen mass is in the order of $0.01 \mathrm{M}_{\odot}$ or less, so the progenitors probably are blue supergiants.

LANGER: Could you give us an indication about which velocities would actually be expected for the $\mathrm{H}$ and $\mathrm{He}$ features?

BRANCH: I can say what velocities are observed: In $\mathrm{SNeIb}$ the minimum detected velocity of hydrogen is about $12000 \mathrm{~km} \mathrm{~s}^{-1}$ and of $\mathrm{He} \mathrm{I}$ is about $7000 \mathrm{~km} \mathrm{~s}^{-1}$. In SNeIc, if hydrogen and helium are present at all, they extend to lower velocities . 